\title{
Ocean Data Assimilation: a case for ensemble optimal interpolation
}

\author{
Peter R. Oke ${ }^{1,2}$, Gary B. Brassington ${ }^{1,3}$, David A. Griffin ${ }^{1,2}$, Andreas Schiller ${ }^{1,2}$ \\ ${ }^{1}$ Centre for Australian Weather and Climate Research \\ A partnership between CSIRO and the Bureau of Meteorology, \\ ${ }^{2}$ CSIRO Wealth from Oceans National Research Flagship, Hobart, Australia \\ ${ }^{3}$ Bureau of Meteorology, Melbourne, Australia
}

Submitted: 4 August 2009

Revised: 15 October 2009

Corresponding author address: P. R. Oke, CSIRO Marine and Atmospheric Research, Castray Esp., GPO Box 1538, Hobart 7001, Tasmania, Australia (peter.oke@csiro.au)

\begin{abstract}
The Bluelink forecast and reanalysis system is comprised of a high resolution ocean general circulation model and an ensemble optimal interpolation (EnOI) system. The Bluelink system has been integrated for a series of 15-year reanalyses and is run operationally at the Bureau of Meteorology to produce short-range ocean forecasts. This system has performed robustly, demonstrating reliable skill that is comparable to other ocean forecast systems around the world. One of the key components of Bluelink is the EnOI system. This system has proved to be a valuable and flexible tool for both operational and research applications. Drawing on Bluelink outcomes and a series of experiments with simple models, a case for using EnOI for ocean data assimilation is made. This includes a discussion of the benefits of EnOI as well as its known limitations. EnOI is robust, flexible, portable, and inexpensive; and is not burdened with the technical difficulties that some other methods carry. EnOI is readily applied for coupled data assimilation and may be an appropriate choice for coupled forecast systems.
\end{abstract}

\section{Introduction}

Bluelink is a partnership between the Commonwealth Scientific and Industrial Research Organisation (CSIRO), the Bureau of Meteorology (BoM) and Royal Australian Navy (RAN). The primary objective of Bluelink is to develop and improve Australia's capabilities in short-range ocean forecasting and reanalysis. The Bluelink forecast system (Brassington et al. 2007) first became operational at the BoM in August 2007, and has since produced two 7-day forecasts each week. The main components of the Bluelink system are the Ocean Forecasting Australia Model (OFAM) and the Bluelink Ocean Data Assimilation System (BODAS; Oke et al. 2008). The primary test-bed for the Bluelink system is the series of Bluelink ReANalysis (BRAN) experiments - multiyear data assimilating model runs (e.g., Schiller et al. 2008; Oke et al. 2009a).

An important aspect of any data assimilation scheme is the estimation of the system's background error covariances. These covariances, together with the observation-error covariances, quantify how the background innovations (model-observation misfits) project onto the full model state - including all variables at all model grid points. Ensemble Kalman Filter (EnKF) methods have been established over many years, with their introduction by Evensen (1994) and subsequent refinement by Burgers et al. 
(1998). Subsequently, many different types of ensemble-based filters have been developed (see Tippett et al. 2003). Ensemble Optimal Interpolation (EnOI), first described by Oke et al. (2002) and Evensen (2003), involves the use of a stationary ensemble of anomalies, or modes, to approximate a system's background error covariances. EnOI has many attractive characteristics, including quasi-dynamically consistent, multivariate, inhomogeneous and anisotropic covariances. The ensemble for EnOI can be time-invariant (e.g., Oke et al. 2002; 2005; 2008; Fu et al. 2008; Counillon and Bertino 2009) or seasonally varying (e.g., Brasseur et al. 2006).

The analysis step of an EnOI scheme is very similar to that of a traditional EnKF, however EnOI is much less expensive. For an application using $m$ ensemble members, EnOI is approximately $m$ times less expensive than an EnKF. EnOI requires only a single deterministic model run to generate a background state, and only a single solution of the analysis equations to update the background. By contrast, an EnKF generally requires the integration of $m$ model runs to represent the time-varying background error covariances and a background state (often based on the ensemble mean), and $m$ solutions of the analysis equations where all ensemble members are updated. Because of its simplicity, EnOI does not represent the errors of the day. Rather, for EnOI, it is assumed that the background error covariances are not state-dependent, and are well represented by a stationary or seasonally varying ensemble. Additionally, EnOI does not explicitly include ensemble forecasts, which have proved useful for quantifying forecast uncertainty.

Several factors, somewhat unique to oceanography, support an argument that EnOI is an appropriate method for ocean data assimilation. These factors are that:

[1] the spatial scales of the most energetic components of ocean variability are short;

[2] ocean observations, particularly observations representing the variability of the interior of the ocean, are sparse; and

[3] the user base for ocean forecasting is small.

The first factor, listed above, means that the spatial resolution of ocean models must be relatively high (e.g., $10 \mathrm{~km}$ or less) to resolve the dominant processes (i.e., eddies). This means that ocean models are generally very computationally expensive, particularly global ocean models. The third factor, listed above, means that justification for the deployment of significant computational resources for ocean forecasting is a challenge. That is, the user demand for ocean forecasting is currently weak. So, with computationally expensive forecast systems motivated by only a small user base, the computational resource available for ocean forecasting is likely to be a limiting factor for the foreseeable future. As a result, optimal data assimilation for ocean applications may not be possible. The second factor together with the first, means that the background, or forecast, errors of ocean forecasts are often quite large, often with entire features (e.g., eddies, meanders, fronts, etc) completely missing in an analysis or forecast. That is, because the observations are sparse and the spatial scales are short, processes and features that are often unpredictable (e.g., instabilities) may not always be either simulated by a model, or detected by the observing system. Taking all of these factors into consideration, one may conclude that an appropriate ocean data assimilation scheme should be computationally inexpensive, robust to missing observations, and capable of introducing, or re-introducing, entire features (e.g., eddies) during any given update. EnOI arguably satisfies all of these criteria. 
Submitted for the special issue of Australian Meteorological and Oceanographic Journal on the proceedings of the CAWCR Workshop on Ensemble Prediction and Data Assimilation 2009

In this paper, the Bluelink forecast system is described, followed by a detailed description of EnOI, a discussion of ocean data assimilation, and some examples of EnOI applied to a global ocean model, followed by a short summary.

\section{Bluelink Forecast System}

The ocean model used under Bluelink is based on the GFDL Modular Ocean Model (Griffies et al., 2004) and is called the Ocean Forecasting Australia Model (OFAM). To date, the developments under Bluelink have focussed on modelling the circulation of the upper ocean in the Australian region. This is reflected in the OFAM grid, with $10 \mathrm{~m}$ vertical grid spacings over the top $200 \mathrm{~m}$, and $1 / 10^{\circ}$ horizontal grid spacings in the $90^{\circ}$ sector centred on Australia and south of $16^{\circ} \mathrm{N}$. The horizontal grid spacing of OFAM is $0.9^{\circ}$ across the Indian and Pacific Ocean and $2^{\circ}$ in the Atlantic Ocean. The Arctic basin is not included in OFAM. To accommodate the inhomogeneous resolution, the horizontal viscosity is resolution and state-dependent based on the Smagorinsky-scheme (Griffies and Hallberg, 2000). The bottom topography for the configuration of OFAM that is used here was constructed from a range of different sources, as documented by Schiller et al. (2008). The turbulence closure model used by OFAM is a version of the hybrid mixed-layer scheme described by Chen et al. (1994).

For long model runs, such as free spin-up runs and BRAN experiments, OFAM is forced by 6-hourly atmospheric fluxes from the European Center for Medium-range Weather Forecasting (ECMWF), using fields from the 40-year ECMWF ReAnalysis (ERA-40; Kallberg et al., 2004) for the period prior to August 2002, and 6-hour operational forecasts thereafter. The operational Bluelink forecast system uses 6-hourly forcing from the BoM Global Atmospheric Prediction System (GASP, e.g., Schulz et al. 2007) that is soon to be replaced with a version of the Unified Model (Rawlins et al. 2007), developed at the UK Met Office.

The Bluelink Ocean Data Assimilation System (BODAS) is described by Oke et al. $(2005 ; 2008)$. BODAS uses an EnOI scheme that is underpinned by a $72-120$ member ensemble (depending on the application) of intraseasonal model anomalies. The ensemble is generated from a long non-assimilating model run. Observations that can be assimilated by BODAS include along-track sea-level anomalies from altimeters, in situ temperature and salinity observations, and satellite sea-surface temperatures.

The Bluelink system has been used to perform operational ocean forecasts (www.bom.gov.au/oceanography/forecasts), multi-year ocean reanalyses (Oke et al. 2005; 2008; 2009a; Schiller et al. 2008), and observing system experiments (Oke and Schiller 2007). Output from Bluelink applications have been used to explore ocean dynamics (e.g., Schiller et al. 2009), for observing system design and assessment (Oke and Schiller 2007; Brassington and Divakaran 2007; Oke et al. 2009b), and to support a variety of industry groups.

\section{Ensemble Optimal Interpolation}

Consider the analysis equations,

$$
\begin{aligned}
& \underline{w}^{a}=\underline{w}^{b}+\underline{K}\left(\underline{d}-\underline{H} \underline{w}^{b}\right), \\
& K=P^{b} H^{T}\left(H P^{b} H^{T}+R\right)^{-1}
\end{aligned}
$$

where $w$ is the model state vector, $d$ is the vector of observations, $K$ is the gain matrix, and $H$ is an operator that maps from model-space to observation-space - often $H$ is simply linear interpolation. The matrix $P$ is the background error covariance and $R$ is 
Submitted for the special issue of Australian Meteorological and Oceanographic Journal on the proceedings of the CAWCR Workshop on Ensemble Prediction and Data Assimilation 2009

the observation error covariance. Superscripts $a$ and $b$ denote analysis and background, respectively. The analysis equations (1-2), can be readily expressed in terms of an ensemble, using

$$
\begin{aligned}
& P^{b}=\rho \circ A^{\prime} A^{\prime T} /(m-1) \\
& K=\rho \circ A^{\prime}\left(H A^{\prime}\right)^{T}\left(\rho \circ H A^{\prime}\left(H A^{\prime}\right)^{T}+(m-1) R\right)^{-1}
\end{aligned}
$$

where $m$ is the ensemble size, $\rho$ is a correlation function, the open circles denote an element-by-element matrix multiplication, and the ensemble $A$, is given by,

$$
A^{\prime}=\alpha\left[\begin{array}{llll}
w_{1}^{\prime} & w_{2}^{\prime} & \cdots & w_{m}^{\prime}
\end{array}\right] \quad \ldots 5
$$

where $\alpha$ is a scalar, and $w_{i}^{\prime}$ is the ith model anomaly. The inclusion of the correlation function in equation (3) and (4) represents localisation (e.g., Houtekamer and Mitchel 2001). Localisation is a necessary part of any ensemble-based data assimilation system where the model state dimension exceeds the ensemble size. Localisation acts to reduce sampling error that arises from the use of a small ensemble, and to increase the rank of the ensemble, so that the system can "fit" the background innovations. One of the negative consequences of localisation is the introduction of dynamical imbalance (e.g., Mitchell et al. 2002; Lorenc 2003; Oke et al. 2007; Kepert 2009). Without localisation (i.e., $\rho=1$ ), it can easily be shown that

$$
w^{a}-w^{b}=A^{\prime} c
$$

where $c$ is a $m$-element vector. Written in this form, it is clear that when an ensemble is used to approximate the background error covariance, the increment, represented by equation (6), is simply a linear combination of ensemble members. When localisation is used, the coefficients $c$, in (6) vary in space - though these coefficients are generally not computed explicitly. This demonstrates that there is a clear relationship between how an ensemble is constructed and the assumptions made when implementing the data assimilation system. This permits a clean hypothesis about the system's background errors. This hypothesis can be readily formulated and evaluated. For example, under Bluelink we hypothesis that the background errors are proportional to model variability on intraseasonal time-scales, relating to the development and evolution of the mesoscale eddy field. The ensemble is therefore constructed in a manner that isolates the representation of the mesoscale eddy field. In practice this is achieved by computing an ensemble of intraseasonal anomalies, through a simple band-pass filter of a long free model run.

The ensemble-based background error covariances generated under EnOI are anisotropic and inhomogeneous. Some examples of ensemble-based correlations are presented in Figure 1. These covariances nicely summarise the dominant processes that regularly occur in different regions. They concisely represent the spatially varying length-scales, anisotropy, and the variance of the modelled circulation. In the examples shown in Figure 1, ensemble-based correlation between sea-level at a reference location (denoted by the star) and sea-level in the surrounding region is shown. These fields show the region of influence of an innovation at the reference location. These examples highlight the long along-shore length-scales and the short across-shore length-scales for a region near the coast (Figure 1a), the anisotropy of the circulation along the path of the EAC after it separates from the coast (Figure 1b), and the quasi-Gaussian, quasiisotropic structures of eddies in a region that is dominated by mesoscale eddies (Figure 1c). 


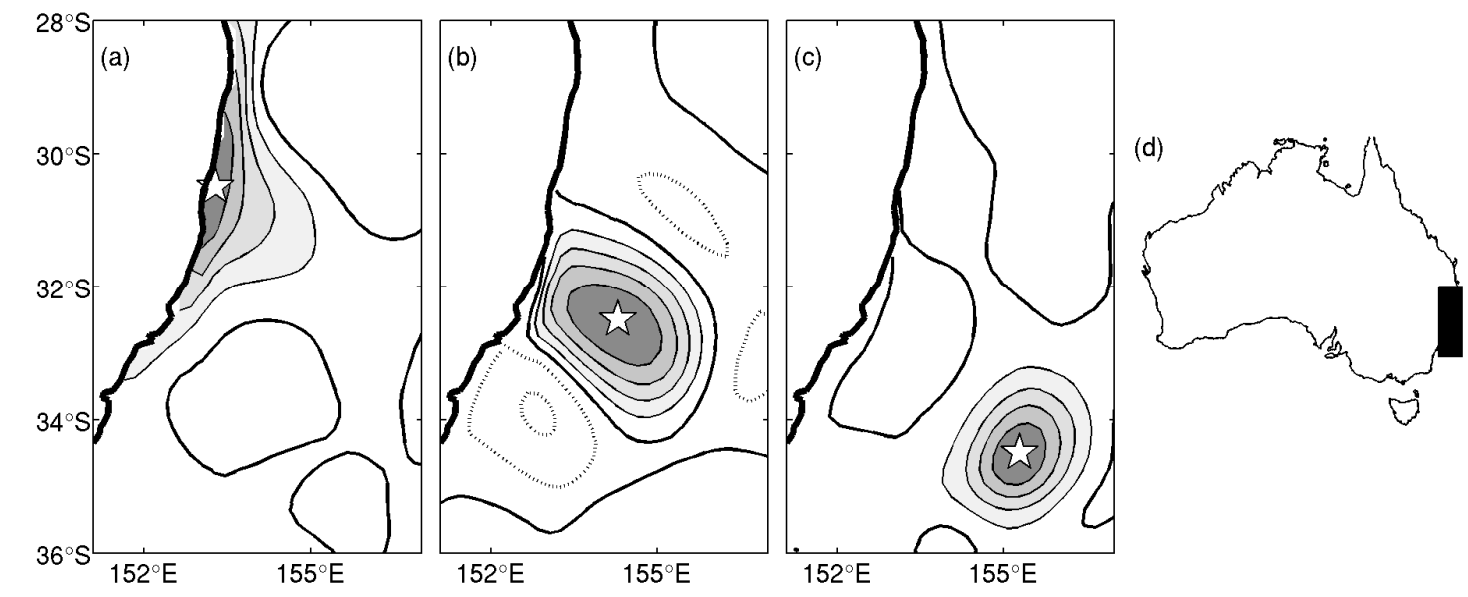

Figure 1: Examples of the ensemble-based cross-correlations between sea-level at a reference location, denoted by the star, and sea-level in the surrounding region for a reference location on the (a) continental shelf, (b) along the path of the EAC as it separates from the coast, and (c) over the deep where EAC-spawned eddies regularly occur off eastern Australia (panel d). Contour intervals are 0.2; zero is bold, dotted is negative, correlations above 0.6 are shaded.

\section{Ocean Data Assimilation}

In the introduction to this paper, there are three aspects of ocean data assimilation that arguably set it apart from atmospheric data assimilation. The first of these is that the spatial length-scales of the ocean are short. The dominance of the short time- and spacescales of the ocean is demonstrated in Figure 2, showing the ratio of the eddy kinetic energy (EKE) to the total kinetic energy (TKE), presented as a percentage, for a 15-year ocean reanalysis (Schiller et al. 2008). This ratio clearly indicates that for much of the ocean, for the region shown, the EKE, representing the short time- and space-scales of the ocean, is significant. In many regions, the EKE completely dominates the variability. For example, the mid-latitudes are dominated by EKE through the abundance of mesoscale eddies (Chelton et al. 2007). There are only a few exceptions to this around Australia, including the transition zones between the equatorial current systems, regions of strong topographic steering (e.g., south of New Zealand) and in some places where persistent continental shelf currents exist (e.g., the Leeuwin Current off Western and Southern Australia).

A sense of the length-scales of the ocean is also obtained by considering a map of sealevel anomaly (Figure 3). This example is typical of the ocean circulation. It shows many relatively small cyclonic and anti-cyclonic features. A time sequence of these fields (not shown; see www.cmar.csiro.au/remotesensing/oceancurrents/) shows that the ocean circulation is very dynamic, with eddies developing over periods of days, and evolving over months. Wave-dynamics are very important in the ocean, with a range of fast- and slow-moving waves representing a considerable proportion of the total ocean variability.

To compare NWP to ocean forecasting, consider the typical length-scales of the ocean relative to the atmosphere. While atmospheric weather patterns span several hundreds and often thousands of kilometres - ocean "weather" patterns typically span 50-300 km. 


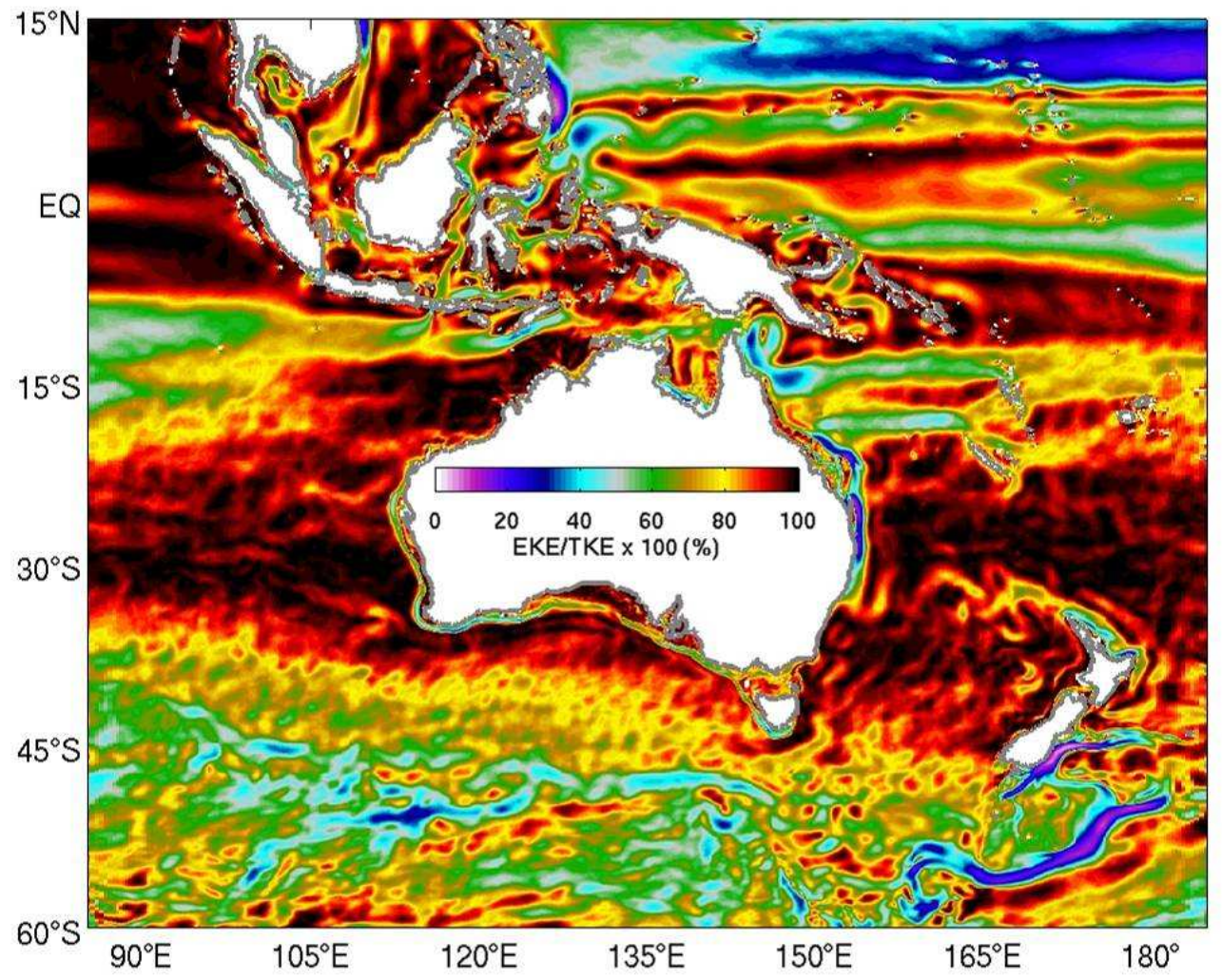

Figure 2: Ratio of eddy kinetic energy to total kinetic energy from BRAN2.1 for the period 1993-2001. Units are in \%.

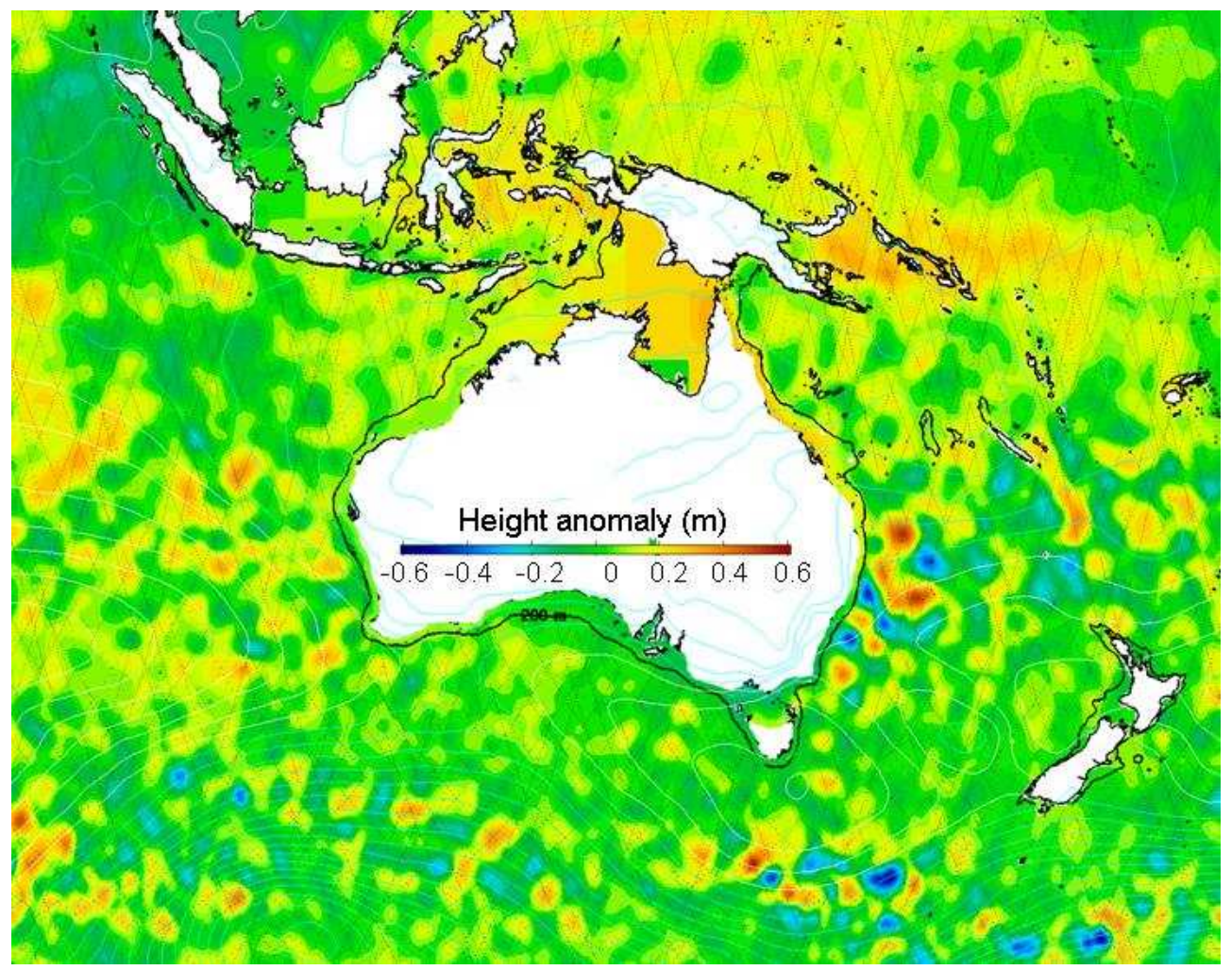

Figure 3: Example of a sea-level anomaly (colour) with atmospheric sea-level pressure overlaid (white and blue contours). The diagonal tracks denote altimeter tracks along which sea-level anomaly is observed, and the black contour shows the $200 \mathrm{~m}$ isobath (sourced from www.cmar.csiro.au/remotesensing/oceancurrents/). 
Consider the status of the global ocean observing system. Conventional ocean observations include sea-level anomaly from satellite altimeters, satellite sea-surface temperature, and in situ observations of sub-surface temperature and salinity profiles from a range of programs (mainly Argo; Argo Science Team, 1998). The typical coverage of the ocean observing system is shown in Figure 4. This includes an example of all observations available on a single day (Figure 4a). Clearly, satellite SST observations provide excellent coverage of the global oceans. These observations are well used by the community and feed through to important applications, like NWP. However, from an oceanographic perspective, SST observations often only represent the variability in the relatively shallow surface boundary layer. Also, in many regions of the ocean SST is isothermal (e.g., a large part of the tropical oceans) and is often somewhat unrelated to the mesoscale variability of the ocean. SST observations are crucial to ocean forecast systems, for their role in initialising and constraining the properties of the upper ocean boundary layer, however, they cannot exclusively constrain the mesoscale variability of the ocean (e.g., Oke and Schiller 2007).

An example of the available sub-surface observations on a single day is presented in Figure $4 \mathrm{~b}$. Here, we have regarded altimeter observations as sub-surface. This is because the observed sea-level anomaly typically represents the properties of the ocean interior in a way that is somewhat analogous to mean sea-level pressure representing the properties of the overlying atmosphere. Sea-level anomaly of the ocean is often highly correlated to variability of the ocean pycnocline that is typically associated with the ocean mesoscale variability. The data distribution in Figure $4 \mathrm{~b}$ provides quite a different story to Figure $4 \mathrm{a}$, indicating that the spatial coverage of sub-surface ocean observations is very sparse compared to the spatial scales of ocean variability (e.g., Figure 3).

Altimeter observations are the most important observation type for constraining the mesoscale eddy field in the ocean (Oke and Schiller 2007; Oke et al. 2009c). The typical repeat-cycle for both satellite altimeters and Argo profiling floats is 10-days. The data coverage over 10-days is presented in Figure 4c. Together with the good SST coverage, the global ocean is relatively well observed over a 10-day period, however even with 10-days of data, the mesoscale eddy field remains under-sampled. Data assimilation systems and ocean analysis systems typically use observations from a timewindow of between 1 and 11 days. Those that use data from only 1-day, attempt to correct the background state only in regions that are observed (e.g., Martin et al. 2007). Those systems that use longer time-windows typically ascribe larger errors to observations that do not correspond to the analysis time, and attempt to reproduce all of the synoptic features of the ocean (e.g., Oke et al. 2008). For both cases, the ocean observing system is likely to miss entire features, like eddies, fronts, or current meanders. If these features are poorly represented in the background field, a subsequent assimilation step may have to introduce entire features. As argued above, and noting the relation presented in equation (6), the ensemble for an EnOI system can readily be constructed to facilitate this. 

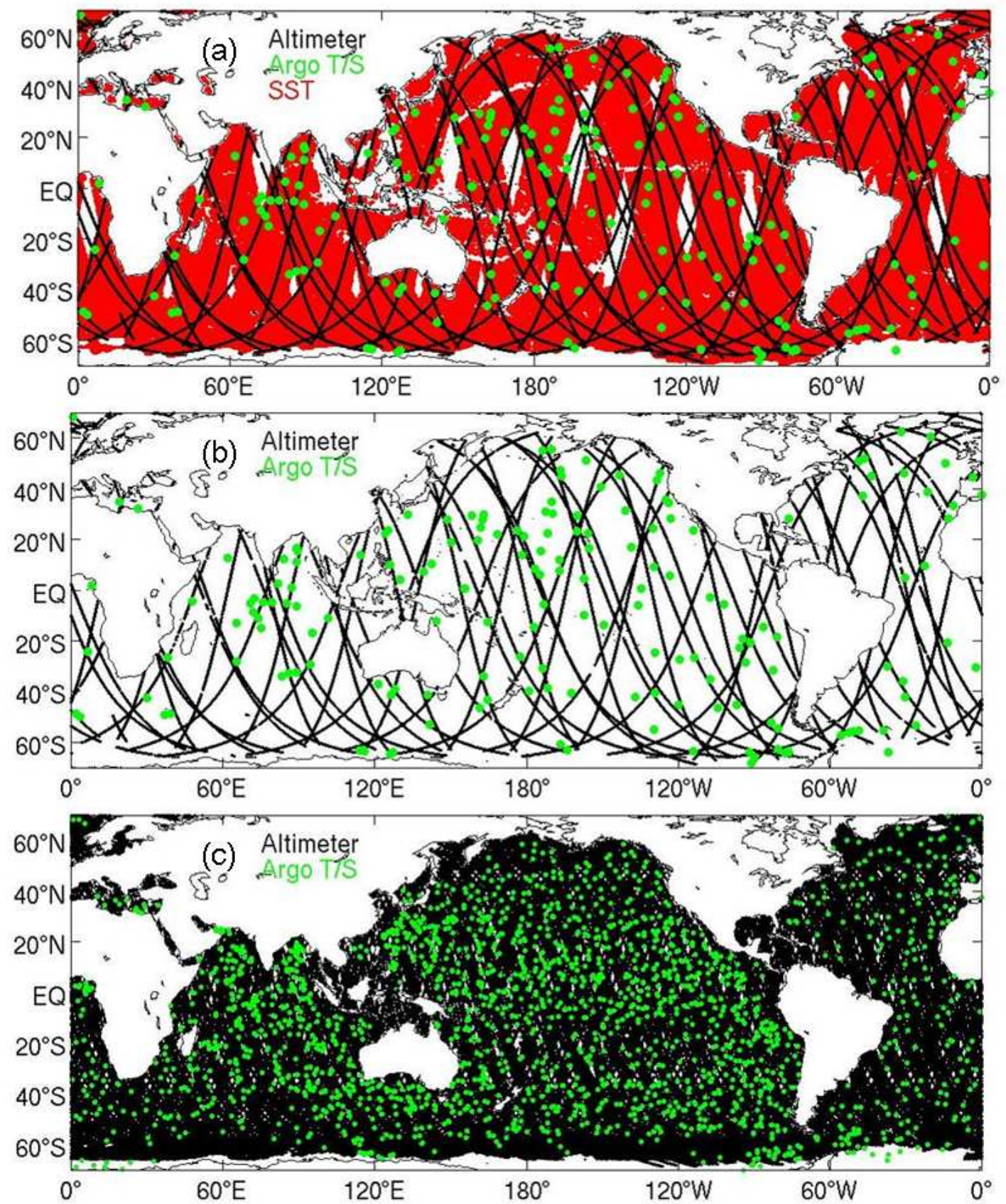

Figure 4: A typical example of observations available in the global ocean observing system showing (a) all observations on a single day, (b) sub-surface observations on a single day, and (c) sub-surface observations for a 10-day window. SST observations (red) typically represent only the shallow surface boundary layer;

Argo T/S (green) are sub-surface profiles of ocean properties; and altimeter observations (black) are here regarded as sub-surface observations because they represent depth-integrals of ocean properties over the upper part of the water column. This example is centred around 1 January 2008. 
Submitted for the special issue of Australian Meteorological and Oceanographic Journal on the proceedings of the CAWCR Workshop on Ensemble Prediction and Data Assimilation 2009

\section{Performance of an EnOI system}

\section{Experiments with small models}

The performance of EnOI and EnKF was compared by Oke et al. (2007) for a simple one-dimensional, linear advection system, following Evensen (2004). Oke et al. (2007) demonstrated that the EnKF is outperforms EnOI, as expected, and investigated the impact of localisation on dynamical balance. The simple system that Oke et al. (2007) used is a perfect advection of a one-dimensional field. They consider experiments where 4 evenly spaced observations are assimilated every 5 time steps over a domain of 1000 grid points. The model state is advected at a constant speed of one grid point per time step (hence "perfect advection"). Both the ensemble and the true field are generated from random samples. For each sample, the variable is initialised as a linear combination of 25 sine curves with random uniform distributed amplitude and phase and a random offset. Thus the dimension of the system is 51. For this configuration, and because the model is linear, it is expected that convergence of the root-mean-squared error is achievable in just under 250 time steps; see Oke et al. (2007) for details.

The performance of EnOI and EnKF for different ensemble sizes, with and without localisation is shown in Figure 5 (adapted from Oke et al. 2007). For this example, the dimension of the system is 51 , so experiments with ensemble sizes of less that 50 require localisation (Figure 5a). This is simply because in these cases the ensemble is rank-deficient - that is, the dimension of the ensemble sub-space is less than the dimension of the model sub-space. For the case with an ensemble size of 100 (i.e., greater than the dimension of the model sub-space), the EnKF out-performs EnOI. For the case with an ensemble size of 50 (i.e., approximately equivalent to the state dimension), the EnKF and EnOI perform comparably. For the cases with an ensemble size of 10 or 20 , without localisation the ensemble is rank-deficient $(<51)$. But for these cases with localisation, provided the localising length-scale is shorter than the distance between observations (here it is 50 and the separation distance is 250), the effective rank of the ensemble becomes 40 or 80 respectively (i.e., number of observations times the ensemble size). So for 10 members with localisation, the ensemble is almost full rank, and for 20 members with localisation, the rank of the ensemble exceeds the dimension of the state.

For the cases with localisation (Figure 5b), all configurations converge on a state that has acceptably small error. Clearly, the EnKF is superior to EnOI - displaying faster convergence - for equivalent ensemble size. In these cases, localisation has acted to increase the rank of the ensemble sufficiently to constrain the system.

The results presented in Figure 5 are not surprising. They clearly indicate that the EnKF outperforms EnOI. However, consider the computational resources required to achieve these results. Recall that the EnKF is $m$-times more expensive that EnOI, where $m$ is the ensemble size. Instead of comparing the EnKF with 100-members and EnOI with 100members, perhaps we should compare the EnKF with 10-members and the EnOI with 100 -members. For these cases, EnOI is still more computationally efficient than the EnKF, requiring significantly less resources, but performs better than the EnKF with and without localisation - in both cases achieving a smaller RMSE. For the case with localisation, the EnKF with 10-members still beats EnOI with 100-members - achieving convergence faster - but the asymptotic skill is better for EnOI. This prompts the question - for an operational system, where computational resources are limited, and where throughput is critical, are we better running an EnKF system with a small dynamic (time-evolving) ensemble, or an EnOI system with a large stationary (time- 
Submitted for the special issue of Australian Meteorological and Oceanographic Journal on the proceedings of the CAWCR Workshop on Ensemble Prediction and Data Assimilation 2009

invariant) ensemble? The answer to this question is unclear, but is something that we think should be pursued.
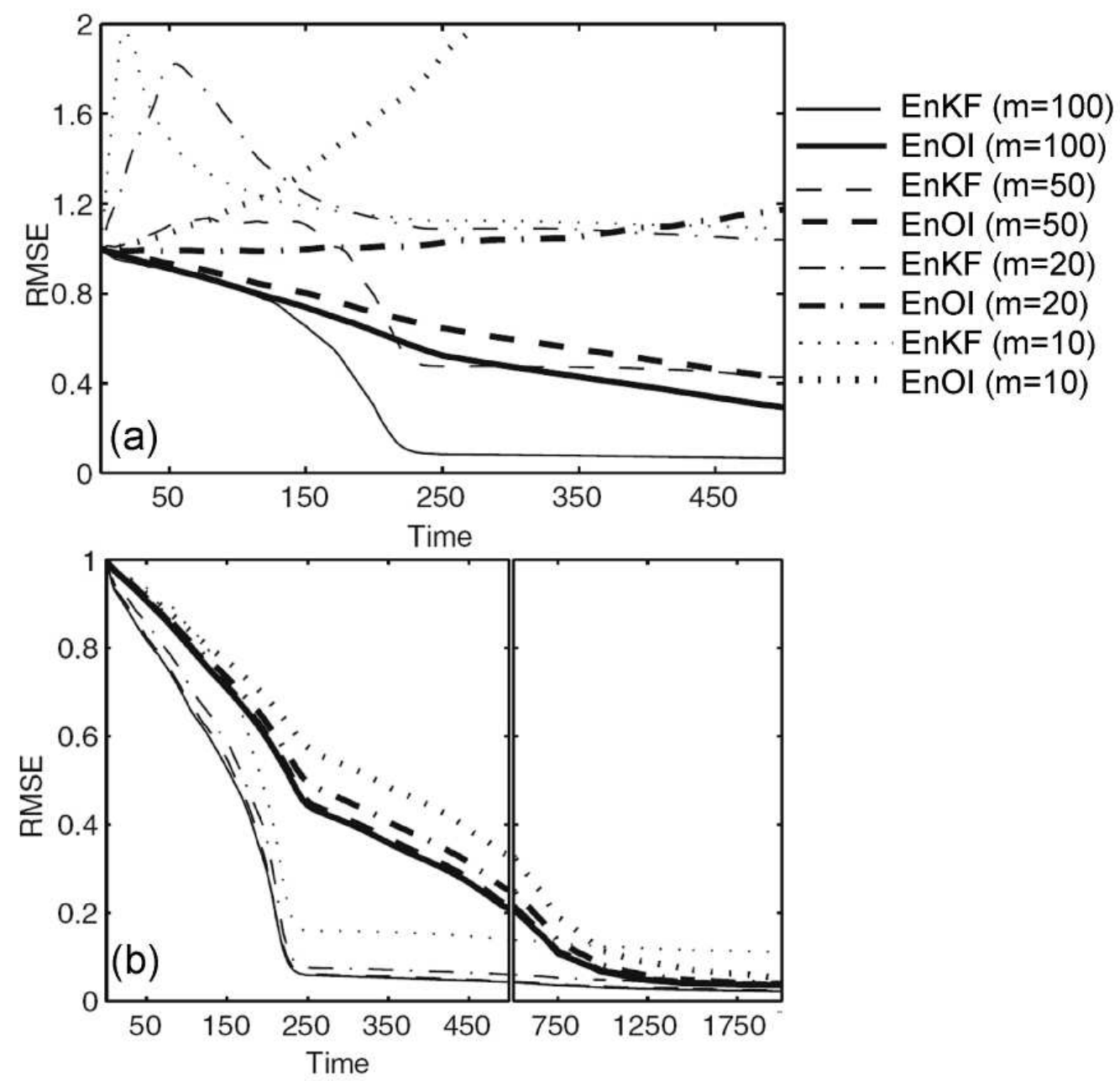

Figure 5: Time series of the root-mean-squared error for an idealised linear advection experiment using an EnKF or EnOI with different ensemble sizes, m, (a) without localisation, and (b) with localisation. Adapted from Oke et al. (2007).

\section{Experiments with general circulation models}

Under Bluelink, an EnOI-based data assimilation system has been used for ocean reanalyses (e.g., Oke et al. 2005; 2008; 2009a; Schiller et al. 2008) and operational ocean forecasting (Brassington et al. 2007). An example of output from the Bluelink system is presented in Figure 6. This example shows monthly mean sea-level fields from BRAN (version 2p2) with drifter-derived velocities and trajectories overlaid. The drifter data are from the entire month. The drifter data represents the time-varying ocean circulation and is therefore a measure of the time-integrated circulation. This is not necessarily well represented by the monthly mean sea-level fields of BRAN. However, provided the variability of the circulation over each month is not too large, this comparison provides an independent assessment of the reanalyzed circulation. Note that data from the surface drifting buoys are not assimilated into BRAN. In general there is good agreement between the drifter trajectories and the sea-level contours, indicating that there is independent agreement between the reanalyzed and observed circulation. 

proceedings of the CAWCR Workshop on Ensemble Prediction and Data Assimilation 2009

The examples in Figure 6 include situations where the drifter trajectories cross the sealevel contours. This is due on occasions to the effects of wind, or may be because a mean field (sea-level) is being compared to a Lagrangian description of the circulation (drifters). It may also be because the mesoscale features reproduced in BRAN are not precisely in the correct positions, or with the correct structures. Nevertheless, this comparison demonstrates that an EnOI system can constrain an ocean general circulation model to reproduce realistic ocean variability.

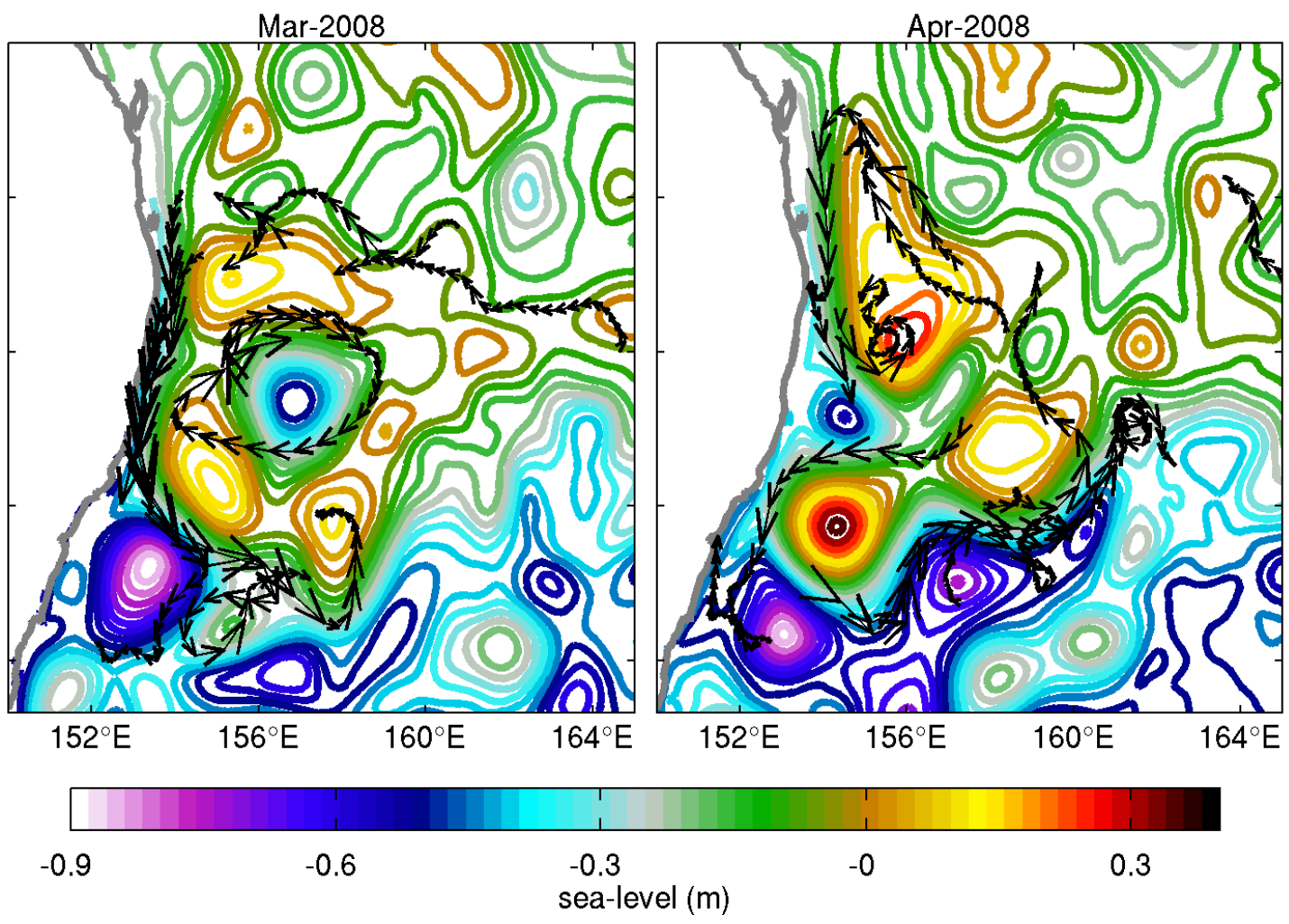

Figure 6: Monthly mean sea-level from BRAN (version 2p2), with surface drifter velocities and trajectories overlaid.

The Global Ocean Data Assimilation Experiment (GODAE), and its successor GODAE OceanView, is an international effort to demonstrate the feasibility of operational ocean forecasting (www.godae.org/). GODAE partners include institutions from Australia, Canada, China, France, Italy, Japan, Norway, UK, and the USA. Most of the systems developed under GODAE perform operational short-range forecasts of the ocean some for specific regions, others for the global ocean. The data assimilation tools used by these systems include EnOI (e.g., Australia, France), multivariate optimal interpolation (MvOI; USA, UK), EnKF (Norway), and variational methods (Japan). Most of these systems are still being developed and improved. To assess the performance of different systems and methods, an inter-comparison exercise was undertaken for a 3-month period in 2008. Some results from this inter-comparison activity are presented below to demonstrate the current state-of-the-art in operational oceanography, and to demonstrate how the EnOI-based systems perform relative to the other operational systems. 
Submitted for the special issue of Australian Meteorological and Oceanographic Journal on the proceedings of the CAWCR Workshop on Ensemble Prediction and Data Assimilation 2009

Here, we compare the performance of systems developed in the USA (HYCOM), France (Mercator), Australia (Bluelink), and the United Kingdom (UKMet). The USHYCOM system is run by the US Navy using a $1 / 12^{\circ}$ resolution global model and an MvOI scheme (Cummings 2005). The French Mercator system uses a global $1 / 4^{\circ}$ resolution model and a SEEK filter implemented with a seasonally-varying ensemble (Brasseur et al. 2006). The UK system (Martin et al. 2007), uses a $1 / 4^{\circ}$ global model and an MvOI based system. Results from Bluelink include BRAN (a delayed-mode reanalysis; Schiller et al. 2008; Oke et al. 2009a), OMAPS-hc (an operational hind-cast; Brassington et al. 2007) and OMAPS-fc (an operational forecast). Of these systems, HYCOM, Mercator, UKMet, and BRAN can each be considered "best estimates". In each case, these are produced using delayed-model atmospheric fluxes and research quality ocean observations. By contrast, OMAPS-hc is an operational hind-cast and OMAPS-fc is an operational forecast, using operational atmospheric fluxes and nearreal-time ocean observations (often with missing and incomplete fields due to data latency).

An example of these inter-comparisons is presented in Figure 7, showing the root-meansquared difference (RMSD) between modelled and observed temperature and salinity for a region off the east coast of Australia. Based on these comparisons it is clear that the EnOI-based systems (i.e., Bluelink and Mercator) perform as well as other groups. A more comprehensive series of inter-comparisons between GODAE systems in the Australian region are available from www.cmar.csiro.au/staff/oke/GODAE.htm. The results presented in Figure 7 are typical. These results of the inter-comparisons performed to date indicate that the majority of the ocean is reasonably well constrained by a stationary, or seasonally varying, ensemble. The likely advantage of an EnKF occurs when and where the time-evolving background error statistics significantly depart from a stationary ensemble estimate, and where a dynamic ensemble has skill at quantifying these time-evolving statistics. We expect that this would occur in regions of energetic currents, such as western boundary currents, or in regions where strong transitions occur, such as strong wind-driven upwellings, or eddy generation. The justification for the additional resources for an EnKF, or other more advanced approaches, should be based on a demonstration of how important these extreme events are to the user community - and more importantly, on how much better an EnKF performs compared to the less expensive EnOI-based systems. 


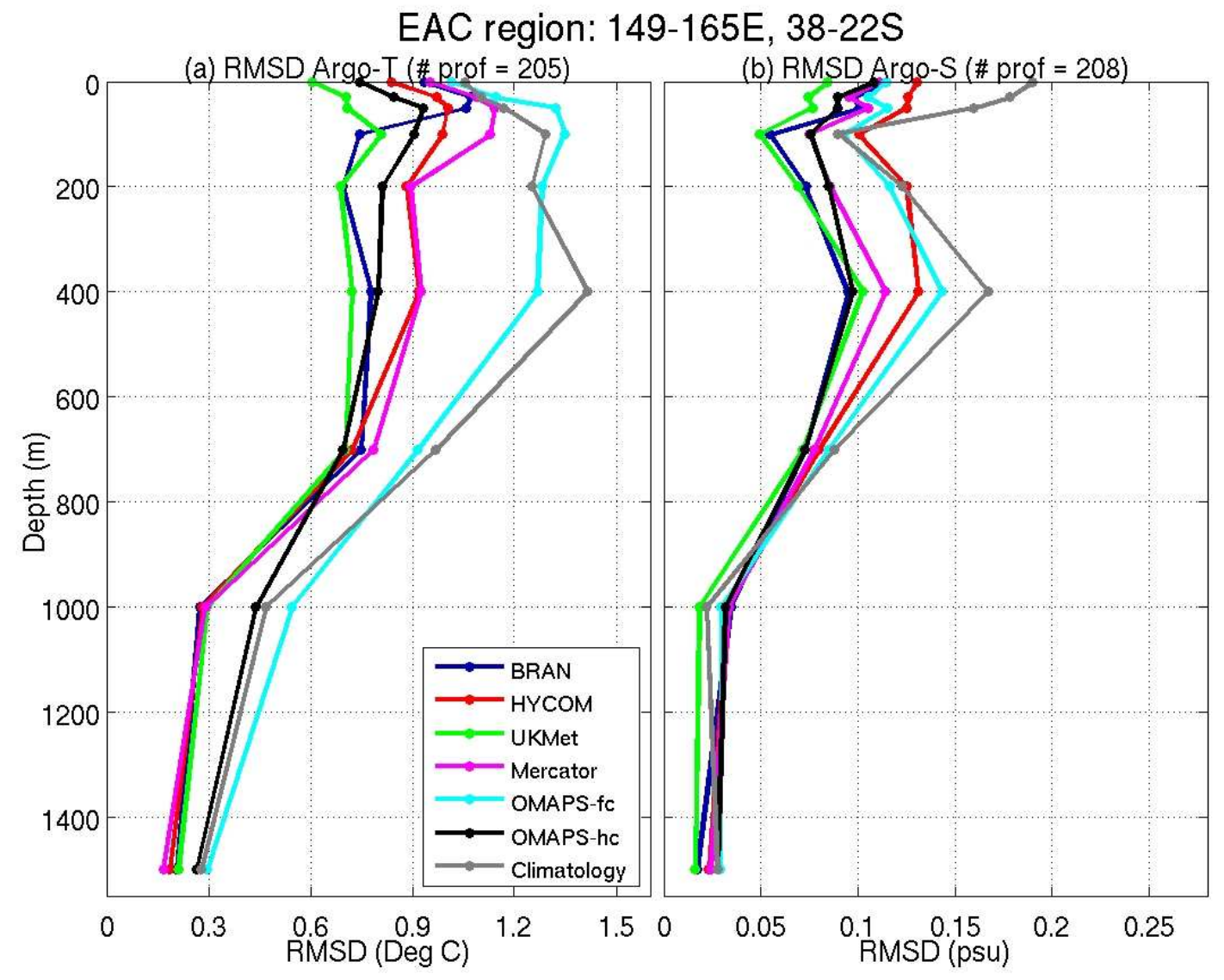

Figure 7: Depth-profiles of the root-mean-squared differences (RMSD) between observed and modelled temperature (left) and salinity (right) from Argo observations and GODAE systems.

\section{Potential extensions}

There is a lot of potential for optimising EnOI-based systems. For example, the extension to a four-dimensional ensemble, where the time-lagged covariances of a system are exploited is relatively straightforward (e.g., Hunt et al. 2004). An example of four-dimensional ensemble-based correlation is presented in Figure 8. This example shows ensemble-based correlations between sea-level from a reference location adjacent to the coast off central eastern Australia at some time $\mathrm{T}=0$, and sea-level in the surrounding region for times $\mathrm{T}=-5, \ldots,+5$. For this example, the ensemble-based correlation indicates that a positive sea-level anomaly at the reference location at the coast is typically preceded by a positive anomaly of sea-level to the south and east (see correlation maps for $\mathrm{T}<0$ ). By contrast, Figure 8 indicates that a positive sea-level anomaly at the reference typically leads a positive sea-level anomaly to the north and a negative anomaly to the east and south-east (see correlation maps for $\mathrm{T}>0$ ). These characteristics are consistent with a Rossby wave, or an eddy, propagating westwards $(\mathrm{T}<0)$ and hitting the coast to the south of the reference location, and generating a northward propagating coastal trapped wave. A dynamical explanation for the negative correlation to the east for $\mathrm{T}>0$ is less clear. So, by using these four-dimensional ensemble-based covariance fields, one could readily produce a four-dimensional analysis that extrapolates the influence of an observation in both time and space in a manner that is analogous to variational representers obtained by adjoint-based assimilation systems (e.g., Bennett 1992). 


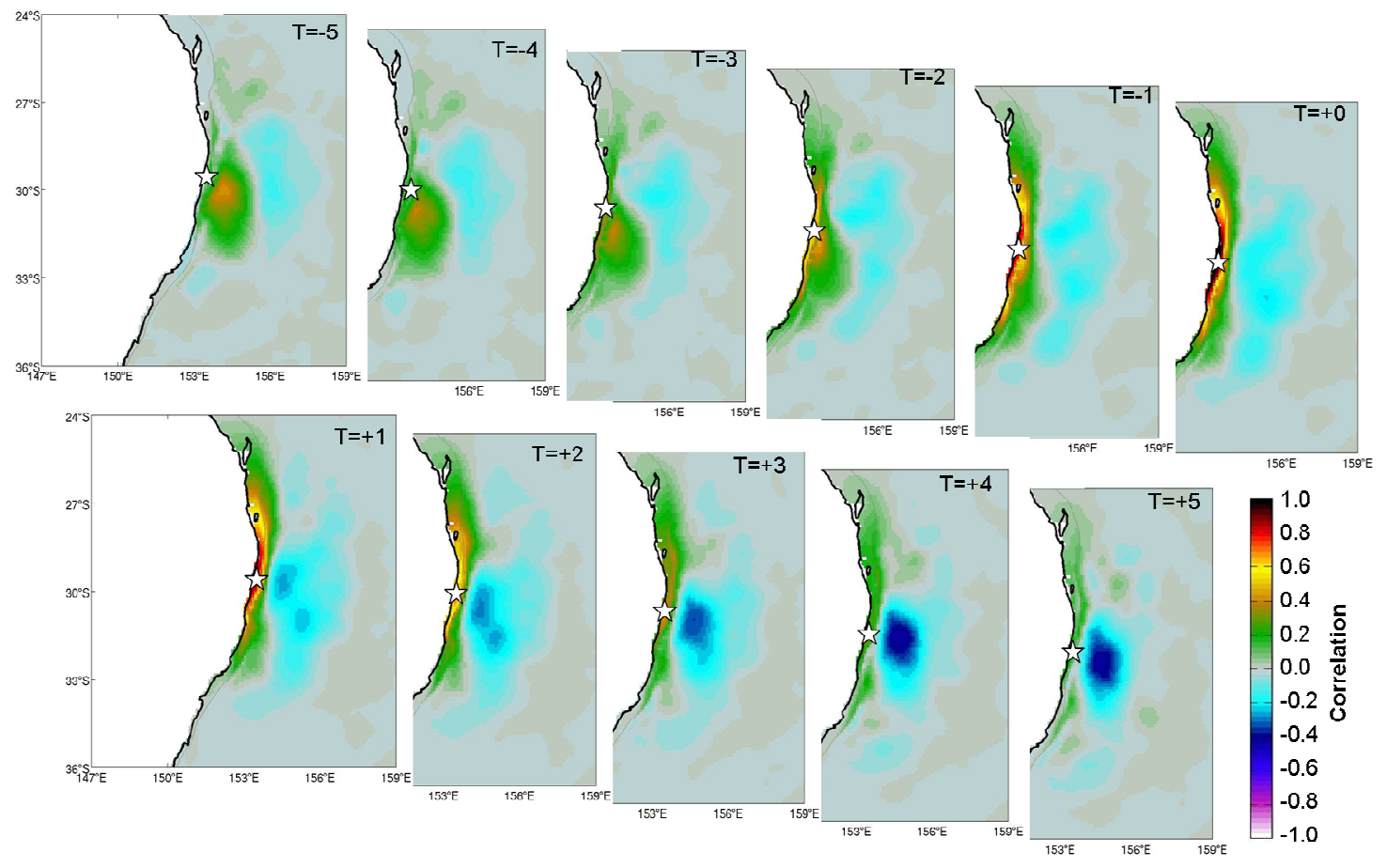

Figure 8: Example four-dimensional ensemble-based correlation maps showing the spatio-temporal influence of a sea-level observation (at the star at $T=0$ ) off the central New South Wales coast.

The extension of EnOI to coupled data assimilation, like the EnKF (e.g., Zhang et al. 2007), is also technically simple. For such applications, the ensemble is augmented by state elements from other systems. Examples of such coupled data assimilation systems could include ocean-atmosphere, ocean-sea-ice, ocean physics-biogeochemistry, and others.

The application of hybrid EnOI-EnKF has been explored by Counillon et al. (2009). They show that the performance of a hybrid EnOI-EnKF system, using 10 dynamic members and 100 stationary members, outperforms both an EnKF with 100 dynamic members and EnOI with 100 stationary members. This option may represent a compromise between the expensive, but potentially optimal EnKF, and the efficient, robust, but sub-optimal EnOI.

EnOI-based systems can be readily used for observing system design and assessment. Oke and Schiller (2007) used the Bluelink EnOI-based system to perform a series of standard observing system experiments to assess the relative importance of different components of the global ocean observing system. Alternatively, methods developed for ensemble square-root filters, together with the stationary ensemble of an EnOI system, can be used to estimate analysis errors obtained by assimilating existing and/or hypothetical observations, as described by Oke et al. (2009b). An example of such an application is presented in Figure 9. This shows the assumed background field (BGF) errors from the Bluelink ensemble - simply the standard deviation of the ensemble and an estimate of the analysis error when a typical array of ocean observations is assimilated. Note that the fields of analysis error for SST are blocky. This is because SST observations are here assumed to be on a fixed grid. Analysis error close to these grid points tends to be smaller than analysis error in between grid points. This example 

proceedings of the CAWCR Workshop on Ensemble Prediction and Data Assimilation 2009

shows the impact of standard ocean observations, but Oke et al. (2009b) also assesses the impact of the assimilation of data from land-based high frequency radar arrays, in situ gliders, and ship-borne T/S sections, at different latitudes along the New South Wales (NSW) coast. Similarly, Brassington and Divikaran (2007) analyse characteristics of an EnOI system to evaluate the potential impact of assimilating seasurface salinity observations on a data assimilation ocean model. These types of studies help evaluate the potential benefits of future observation programs on ocean forecast and reanalysis systems.

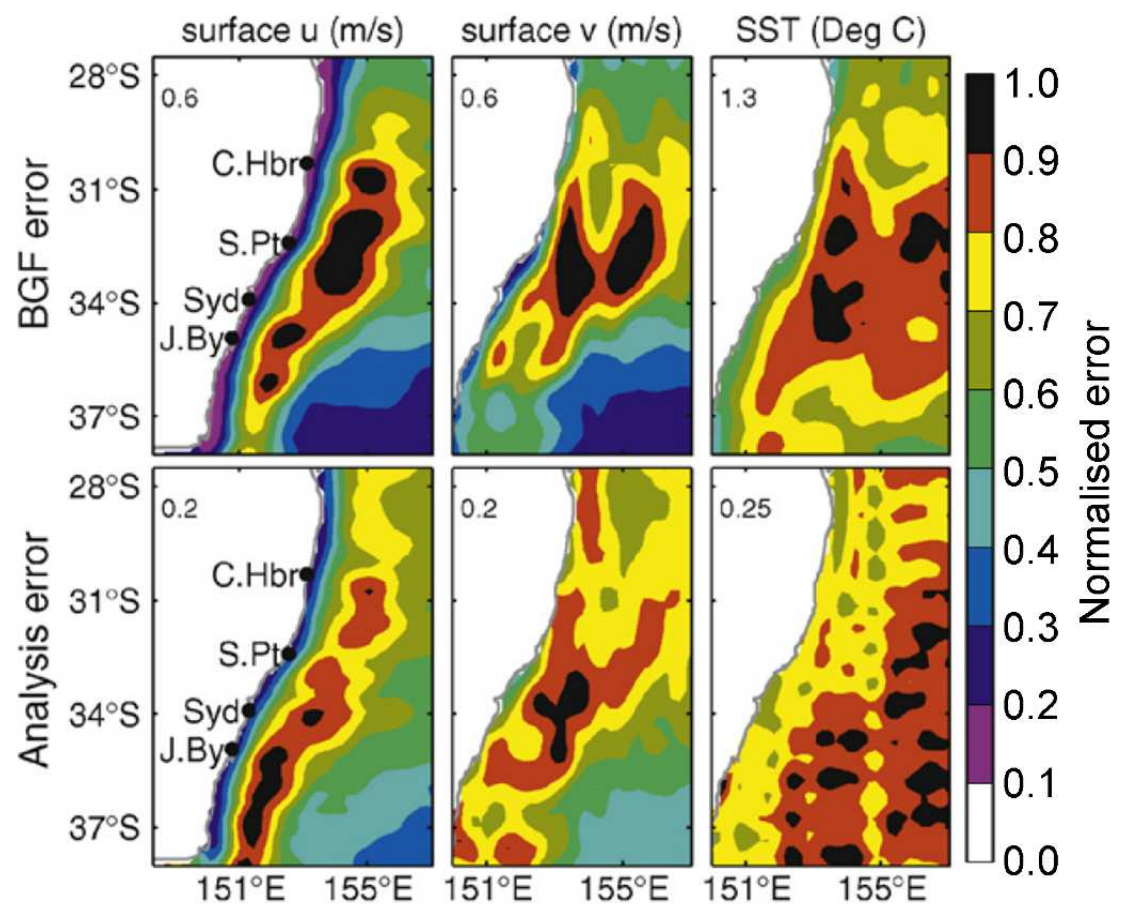

Figure 9: Estimates of the background field (BGF) error (top) and the analysis error when observations of sea-level anomaly, SST, and Argo T/S are assimilated (bottom) for the Bluelink system for variables surface velocities ( $u$ and v), and SST (left-right). The legend shows the normalised error. Each panel is normalised by the number shown in the top left corner of each panel. Adapted from Oke et al.

(2009b).

\section{Conclusion}

The performance of an EnKF and EnOI system was compared by Oke et al. (2007) for a simple linear advection system. They showed that while EnKF outperforms EnOI, EnOI is a robust system that can produce results of comparable performance to the EnKF. Based on their results, and on considerations of the relative computational cost of EnKF compared to EnOI, we have here argued that for some applications it may be worth considering running an EnOI system with a large stationary ensemble, rather than an EnKF system with a small dynamic ensemble.

To date the application of EnOI to realistic global systems is limited to the Bluelink and Mercator systems, under GODAE OceanView. Bluelink uses a stationary ensemble, while Mercator uses a seasonally-varying ensemble of anomalies, or modes, to approximate the system's background error covariances. Comparisons between the performance of these EnOI-based systems and other operational ocean forecasting systems demonstrate that they produce results that compare well to other methods. 
Submitted for the special issue of Australian Meteorological and Oceanographic Journal on the proceedings of the CAWCR Workshop on Ensemble Prediction and Data Assimilation 2009

Preliminary investigations into the potential of a hybrid EnOI-EnKF, where a small dynamic ensemble is supplemented by a larger stationary ensemble show promising performance (Counillon et al. 2009).

In this paper we present a series of arguments to make a case for using EnOI for ocean data assimilation. Specifically, we refer to the dominance of short spatial scales of the ocean and the relative sparseness of the ocean observing system. Together, these factors mean that an eddy-resolving, data assimilation ocean model is likely to miss entire features, such as eddies or fronts, that are not always represented by observations or forecasts. We note that EnOI is particularly well suited to introducing new dynamical features, because of the user's control over the types of features and scales represented by a stationary ensemble. We also refer to the relationship between the ensemble anomalies, or modes, and the increments. That is, that the increments are a linear combination of ensemble anomalies. This recognition provides guidance for how an ensemble should be generated - and allows the user to make and test hypotheses about the system's background errors. Together, we present these arguments as justification for why EnOI may be a good choice for ocean data assimilation.

Finally, we note the natural extensions of EnOI. These include an extension to fourdimensional analyses, using a time-lagged ensemble, and to coupled data assimilation, where the ensemble is augmented with fields from coupled systems (e.g., atmosphereocean). We also demonstrate the application of EnOI to objective observing system assessment and design.

\section{Acknowledgements}

Financial support for this research is provided by CSIRO, the Bureau of Meteorology, and the Royal Australian Navy as part of the Bluelink project, and the US Office of Naval Research (Grant No. N00014-07-1-0422). The author also acknowledges the many contributions of the Bluelink science team and the comments of two anonymous reviewers, Dr. J. Kepert and Dr. S. Marsland I suspect. Satellite altimetry is provided by NASA, NOAA, ESA and CNES. Drifter data are provided by NOAA-AOML and SST observations are provided by NASA, NOAA and Remote Sensing Systems.

\section{References}

Argo Science Team, 1998: On the design and implementation of Argo: An initial plan for a global array of profiling floats. International CLIVAR Project Office Rep. 21, GODAE Rep. 5, GODAE Project Office, Melbourne, Australia, 32 pp.

Bennett, A. F., 1992: Inverse Methods in Physical Oceanography. Cambridge University Press, 346 pp.

Brasseur, P., Bahurel, P., Bertino, L., Birol, F., Brankart, J.-M., Ferry, N., Losa, S., Remy, E., Schrter, J., Skachko, S., Testut, C.-E., Tranchant, van Leeuwen, P.-J., Verron, J. 2006: Data assimilation in operational ocean forecasting systems: the MERCATOR and MERSEA developments. Quarterly Journal of the Royal Meterological Society, 131, 3561-3582.

Brassington, G. B., Pugh, T. F., Spillman, C., Schulz, E., Beggs, H., Schiller, A., Oke, P. R., 2007: BLUElink> development of operational oceanography and servicing in Australia. Journal of Research Practice Information Technology, 39, 151164. 
Submitted for the special issue of Australian Meteorological and Oceanographic Journal on the proceedings of the CAWCR Workshop on Ensemble Prediction and Data Assimilation 2009

Brassington, G. B., Divakaran, P., 2008: The theoretical impact of remotely sensed sea surface salinity observations in a multi-variate assimilation system, Ocean Modelling, 27, 70-81 10.1016/j.ocemod.2008.12.005

Burgers, G., van Leeuwen, P. J., Evensen, G., 1998: Analysis scheme in the ensemble Kalman filter. Mon. Wea. Rev., 126, 1719-1724.

Chelton, D. B., Schlax, M. G., Samelson, R. M., de Szoeke, R. A., 2007: Global Observations of Large Oceanic Eddies. Geophys. Res. Lett., 34, L15606, doi:10.1029/2007GL030812.

Counillon, F., Bertino, L., 2009: Ensemble Optimal Interpolation: multivariate properties in the Gulf of Mexico. Tellus-A, 61, $296-308$.

Counillon, F., Sakov, P., Bertino, L., 2009: Application of a hybrid EnKF-OI to ocean forecasting. Ocean Dynamics, submitted.

Evensen, G., 1994: Sequential data assimilation with a nonlinear quasi-geostrophic model using Monte Carlo methods to forecast error statistics. J. Geophys. Res., 99, $10143-10162$.

Evensen, G., 2003. The Ensemble Kalman Filter: theoretical formulation and practical implementation. Ocean Dyn., 53, 343-367.

Evensen G., 2004. Sampling strategies and square root analysis schemes for the EnKF. Ocean. Dyn., 54, 539-560

Fu W., Zhu, J., Yan, C., 2008. A comparison between 3DVAR and EnOI techniques for satellite altimetry data assimilation. Ocean Modelling, 26, 206-216.

Houtekamer, P. L., Mitchell, H. L., 2001. A sequential ensemble Kalman filter for atmospheric data assimilation. Mon. Weath. Rev., 129, 123-137.

Hunt, B. R., Kalnay, E., Kostelich, E. J., Ott, E., Patil, D. J, Sauer, T., Szunyogh, I., Yorke, J. A., Zimin, A. V., 2004. Four-dimensional ensemble Kalman filtering. Tellus, 56A, 273-277.

Kepert, J.D., 2008. Covariance localisation and balance in an ensemble Kalman filter. Quart. J. R. Meteor. Soc., 135, 1157-1176.

Lorenc A. C., 2003. The potential of the ensemble Kalman filter for NWP - a comparison with 4D-Var. Quart. J. Roy. Meteor. Soc., 129, 3183-3203.

Martin, M. J., Hines, A., Bell, M. J., 2007. Data assimilation in the FOAM operational short-range ocean forecasting system: a description of the scheme and its impact. Quart. J. R. Meteor. Soc., 133, 981-995.

Mitchell, H. L., Houtekamer, P. L., Pellerin, G., 2002. Ensemble size, balance, and model-error representation in an ensemble Kalman filter. Mon. Weath. Rev., 130, 2791-2808.

Oke, P. R., Allen, J. S., Miller, R. N., Egbert, G. D., Kosro, P. M., 2002. Assimilation of surface velocity data into a primitive equation coastal ocean model. J. Geophys. Res., 107, 3122. doi:10.1029/2000JC000511.

Oke, P. R., Schiller, A., Griffin, D. A., Brassington, G. B., 2005. Ensemble data assimilation for an eddy-resolving ocean model of the Australian Region. Quart. J. R. Meteor. Soc., 131, 3301-3311. 
Submitted for the special issue of Australian Meteorological and Oceanographic Journal on the proceedings of the CAWCR Workshop on Ensemble Prediction and Data Assimilation 2009

Oke, P. R., Sakov, P., Corney, S. P., 2007. Impacts of localisation in the EnKF and EnOI: experiments with a small model. Ocean Dyn., 57, 32-45.

Oke, P. R., A. Schiller. 2007. Impact of Argo, SST and altimeter data on an eddyresolving ocean reanalysis. Geophysical Research Letters, 34(L19601): doi:10.1029/2007GL031549.

Oke, P. R., Brassington, G. B., Griffin, D. A., Schiller, A., 2008. The Bluelink Ocean Data Assimilation System (BODAS), Ocean Modelling, 21, 46-70, doi:10.1016/j.ocemod.2007.11.002.

Oke, P. R., Brassington, G. B., Griffin, D. A., Schiller, A., 2009a. Data assimilation in the Australian Bluelink system. Mercator Ocean Quarterly Newsletter, 34, 3544.

Oke, P. R., Sakov, P., Schulz, E., 2009b: A comparison of shelf observation platforms for assimilation into an eddy-resolving ocean model. Dynamics of Atmospheres and Oceans, 48, 121-142, doi:10.1016/j.dynatmoce.2009.04.002.

Oke, P. R., Balmaseda, M. A., Benkiran, M., Cummings, J. A., Dombrowsky, E., Fujii, Y., Guinehut, S., Larnicol, G., Le Traon, P.-Y., Martin, M. J., 2009c. Observing System Evaluations using GODAE systems. Oceanography, 22(3), 144-153.

Schiller, A., Oke, P. R., Brassington, G. B., Entel, M., Fiedler, R., Griffin, D. A., Mansbridge, J. V., 2008. Eddy-resolving ocean circulation in the AsianAustralian region inferred from an ocean reanalysis effort. Progress in Oceanography, 76, 334-365.

Schiller, A., Ridgway, K. R., Steinberg, C. R., Oke, P. R., 2009. Dynamics of Three Anomalous SST Events in the Coral Sea. Geophysical Research Letters, 36, L06606, doi:10.1029/2008GL036997.

Tippett, M. K., Anderson, J. L., Bishop, C. H., Hamill, T. M., Whitaker, J. S., 2003. Ensemble square root filters. Mon. Wea. Rev., 131, 1485-1490.

Zhang, S., Harrison, M. J., Rosati, A., Wittenberg, A., 2007. System Design and Evaluation of Coupled Ensemble Data Assimilation for Global Oceanic Climate Studies. Mon. Weath. Rev., 135, 3541-3564. 